\title{
Respiratory morbidity of preterm infants of less than 33 weeks gestation without bronchopulmonary dysplasia: a 12-month follow-up of the CASTOR study cohort
}

\author{
B. FAUROUX ${ }^{1 *}$,J.-B. GOUYON ${ }^{2}$, J.-C. ROZE ${ }^{3}$, C. GUILLERMET-FROMENTIN ${ }^{4}$, \\ I. GLORIEUX ${ }^{5}$, L. ADAMON ${ }^{6}$, M. DI MAIO ${ }^{7}$, D. ANGHELESCU ${ }^{8}$, \\ T. MILORADOVICH ${ }^{8}$, B. ESCANDE ${ }^{9}$, C. ELLEAU ${ }^{10}$ AND D. PINQUIER ${ }^{11}$ \\ ${ }^{1}$ Armand Trousseau Hospital, Paediatric Pulmonary Department, Research Unit INSERM U 955, National \\ Reference Centre for Rare Lung Diseases, Pierre et Marie Curie University Paris 6, France; ${ }^{2}$ Reunion University \\ Hospital-Hospital Group South; Centre of Perinatal Studies of Indian Ocean, Reunion Island, France; \\ ${ }^{3}$ Department of Neonatology, Nantes University Hospital, France; ${ }^{4}$ Department of Paediatric Intensive Care, \\ Besançon University Hospital, France; ${ }^{5}$ Department of Neonatology, Toulouse University Hospital, France; \\ ${ }^{6}$ Department of Neonatology, Caen University Hospital, France; ${ }^{7}$ Department of Paediatrics and Neonatology, \\ Nimes University Hospital, France; ${ }^{8}$ AbbVie France; ${ }^{9}$ Department of Neonatal Intensive Care, Strasbourg \\ University Hospital, France; ${ }^{10}$ Department of Paediatrics, Bordeaux University Hospital, France; \\ ${ }^{11}$ Department of Neonatology, Rouen University Hospital, France
}

Received 21 December 2012; Final revision 24 April 2013; Accepted 3 July 2013; first published online 13 September 2013

\section{SUMMARY}

The aim of this study was to describe the incidence and risk factors for respiratory morbidity during the 12-month period following the first respiratory syncytial virus (RSV) season in 242 preterm infants $[<33$ weeks gestational age (GA)] without bronchopulmonary dysplasia and 201 full-term infants (39-41 weeks GA) from the French CASTOR study cohort. Preterm infants had increased respiratory morbidity during the follow-up period compared to full-terms; they were more likely to have wheezing $(21 \%$ vs. $11 \%, P=0.007)$ and recurrent wheezing episodes ( $4 \%$ vs. $1 \%, P=0 \cdot 049$ ). The 17 infants (14 preterms, three full-terms) who had been hospitalized for RSV-confirmed bronchiolitis during their first RSV season had significantly more wheezing episodes during the follow-up period than subjects who had not been hospitalized for RSVconfirmed bronchiolitis (odds ratio $4 \cdot 72,95 \%$ confidence interval $1 \cdot 71-13 \cdot 08, P=0 \cdot 003$ ). Male gender, birth weight $<3330 \mathrm{~g}$ and hospitalization for RSV bronchiolitis during the infant's first RSV season were independent risk factors for the development of wheezing episodes during the subsequent 12-month follow-up period.

Key words: Asthma, bronchiolitis, hospitalization, morbidity, premature infant, respiratory syncytial virus (RSV), risk factor, wheezing.

\section{INTRODUCTION}

Bronchiolitis is the most common seasonal viral lower respiratory tract infection (LRTI) in infancy [1-3].

\footnotetext{
* Author for correspondence: Professor B. Fauroux, Paediatric Pulmonary Department and INSERM U 955, National Reference Centre for Rare Lung Diseases, Pierre et Marie Curie-Paris 6 University, AP-HP, Armand Trousseau Hospital, 26 avenue du Docteur Arnold Netter, 75012 Paris, France. (Email: brigitte.fauroux@trs.aphp.fr).
}

The leading aetiological agent of bronchiolitis is respiratory syncytial virus (RSV) that accounts for up to $80 \%$ of cases in infants during their first epidemic season [1-5]. Although $<2 \%$ of infants with bronchiolitis require hospital admission, RSV is responsible for up to $75 \%$ of the hospitalizations for severe bronchiolitis in infants aged $<1$ year $[2,4,6]$.

There is substantial epidemiological evidence supporting the concept that severe RSV bronchiolitis

The online version of this article is published within an Open Access environment subject to the conditions of the Creative Commons Attribution-NonCommercial-No Derivatives licence $<$ http://creativecommons.org/licenses/by-nc-nd/3.0/ $>$. The written permission of Cambridge University Press must be obtained for commercial re-use. 
resulting in hospitalization and occurring in the first months of life is associated with recurrent wheezing or asthma during childhood [7-9]. A recent literature review, which pooled data from epidemiological studies on the impact of severe RSV bronchiolitis on the subsequent respiratory morbidity, concluded that the history of severe bronchiolitis is strongly associated with the development of recurrent wheezing throughout childhood, and speculated on a complex relationship between viral LRTI in early life and the development of bronchial hyperreactivity [10]. In the Tucson Children's Respiratory Study, early RSV LRTI was an independent risk factor of subsequent wheezing up to age 11 years but ceased to be so at age 13 years [11]. Sigurs and colleagues demonstrated an increased prevalence of asthma or recurrent wheezing and allergic sensitization in a cohort of infants hospitalized with RSV bronchiolitis during the first year of life compared to a matched control cohort at ages 3, 7 and 13 years [12-14]. Recently, the same group showed that severe RSV bronchiolitis, requiring hospitalization during the first year of life, is frequently followed by allergic asthma persisting into early adulthood [15], contrasting with previous data suggesting a shorter post-RSV bronchial hyperresponsiveness [16-18]. Conversely, asthma was associated with a threefold long-term increased susceptibility for severe RSV disease, suggesting that some host factors could be responsible for the severe response to RSV infection [16].

These data favour the hypothesis of a common genetic predisposition and/or common environmental exposures underlying the propensity of severe RSV infection and asthma rather than a causal effect of RSV [16, 17]; RSV bronchiolitis might not be a trigger for subsequent asthma but simply a marker of the asthmatic genetic predisposition [18].

A number of recent studies have identified respiratory viruses other than RSV that might be involved in bronchiolitis, recurrent wheezing and asthma [17, 19-25]. About 10-20\% of bronchiolitis cases are due to rhinovirus (hRV), parainfluenza, influenza, adenovirus or human metapneumovirus (hMPV) [25]. Valkonen et al. [24] showed that infants hospitalized with non-RSV bronchiolitis have a substantially higher risk of developing recurrent wheezing during a 3-year period than those with RSV bronchiolitis. The Childhood Origins of Asthma (COAST) study also highlighted a close association between hRV infection and subsequent wheezing in a cohort of children at high risk of developing asthma or allergy [19].
Conversely, influenza virus infection was not associated with an excess of asthma episodes [26]. The relationships between atopy, early viral respiratory infections, clinical bronchiolitis, and the development of asthma are rather complex, and further prospective studies are warranted to provide a better understanding of the underlying mechanisms [27].

Chronic respiratory morbidity is common after premature birth; in premature infants, the immaturity of the immune system confers an increased risk for severe respiratory viral infections and airway obstruction in the first 2 years of life [28-32]. Prematurity with respiratory morbidity, such as respiratory distress syndrome, can result in long-term lung damage and bronchial hyperreactivity, which predisposes to subsequent viral-induced wheezing later on in life [33]. Romero et al. [34] showed that early RSV LRTIs in preterm infants were associated with an increased prevalence of serious early childhood wheezing by age 3 years. Moreover, it has been shown that RSV and hMPV LRTIs in preterm infants are associated with abnormal lung function and more wheezing at follow-up, persisting in adolescents and young adults, especially in those with a history of severe bronchopulmonary dysplasia (BPD) $[35,36]$.

After the CASTOR study in which we showed that prematurity of $<33$ weeks gestational age (GA) was the only factor associated with a significantly increased risk of hospitalization for bronchiolitis in non-BPD infants during the infants' first RSV season, we performed this follow-up study of the same cohort, in order to evaluate the respiratory morbidity of these premature infants during the subsequent 12-month period. The aims of this follow-up study were first to describe the respiratory morbidity of the premature and full term infants, and second to identify the risk factors for respiratory morbidity during the 12-month period following the first RSV season.

\section{METHODS}

\section{Design of the CASTOR study and its respiratory follow-up}

This study was a follow-up study of the CASTOR study, and is referred to as the CASTOR follow-up study. The (original) CASTOR study was a multicentre, ambispective, longitudinal, non-interventional, epidemiological study conducted to compare hospitalization rates for RSV-confirmed bronchiolitis between preterm infants $<33$ weeks GA without BPD and 


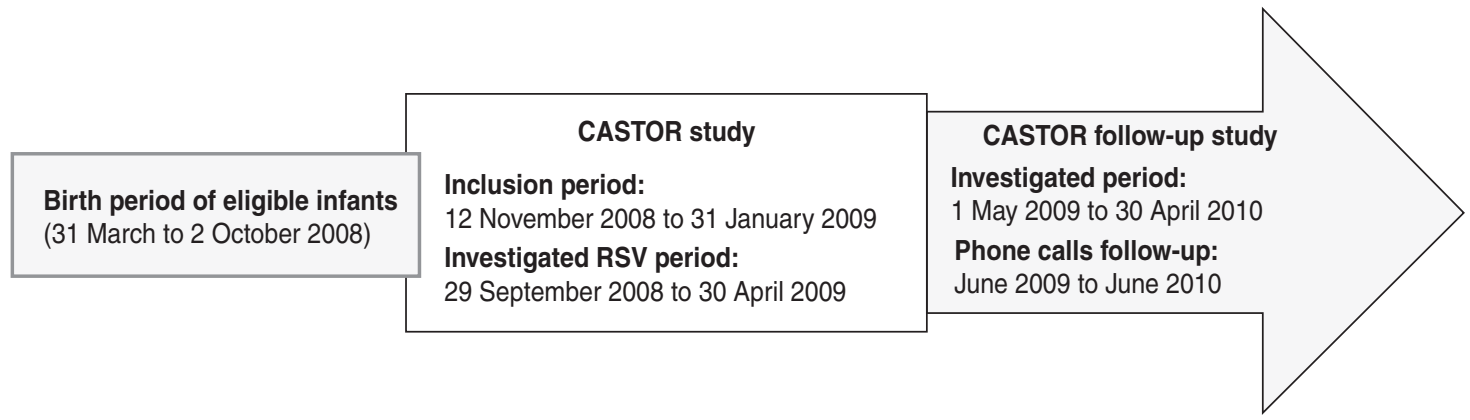

Fig. 1. Study design.

matched full-term infants followed up during the 2008-2009 RSV season in France.

The rationale, design and outcomes of the CASTOR study have been presented in detail in a previous article [37]. In brief, 548 infants were recruited in nine regional neonatology networks (types 2 and 3 hospital centres) from 12 November 2008 to 31 January 2009. Each participating physician identified all the preterm infants $<33$ weeks GA without BPD born between 31 March and 2 October 2008 and matched each preterm to a full-term infant, according to chronological age \pm 1 month, gender and birth region; a total of 498 infants were matched (249 in each group). This cohort was followed during the 2008-2009 RSV season (Fig. 1); data on bronchiolitis hospitalizations from 29 September 2008 to inclusion (i.e. 12 November 2008) were collected retrospectively, and those regarding the hospitalizations from inclusion to the end of the study (i.e. 30 April 2009) were collected prospectively. Parents were telephoned every month; during the last call scheduled in the CASTOR study, they were asked whether they would participate in the CASTOR follow-up study during the next 12 months.

\section{Study population of the CASTOR follow-up study}

Eligible infants for the CASTOR follow-up study were those included in the CASTOR study, i.e. with chronological age $<6$ months upon CASTOR study enrolment, not having received specific RSV prophylaxis, and without documented immune deficiency or other serious or chronic illness that may have had an impact on their health status at the beginning of the 2008-2009 RSV season.

Infants with a GA $<33$ weeks without BPD (defined as oxygen dependence at 28 days of life) were eligible as preterm infants, and those with a GA between 39 and 41 weeks as full-term infants.

\section{Data collection}

During quarterly phone calls performed between June 2009 and June 2010, parents were asked whether their child had been hospitalized for bronchiolitis, wheezing, asthma or other respiratory morbidity, and whether the child had any wheezing episodes or any other episode of non-specified respiratory morbidity (NSRM, defined as any episode of respiratory morbidity reported by parents, excluding bronchiolitis, wheezing, asthma).

Data were collected for the period from 1 May 2009 to 30 April 2010. Parents were supplied with a diary to help them remember hospitalization episodes and identify the practitioners in charge of these hospitalizations. All these data were reported via a parents' questionnaire. Between phone calls, parents had a toll-free number at their disposal to report any hospital admission for bronchiolitis. Physicians in charge of hospitalizations had to complete a hospitalization form for each child that requested medical data related to any hospitalizations for bronchiolitis (RSV confirmed or not) or other respiratory problems occurring during the 12-month follow-up period starting 1 May 2009.

\section{Statistical analysis}

The statistical analyses were performed using SAS software, version 9.2 (SAS Institute Inc., USA). Quantitative variables were described by their frequency, mean plus or minus standard deviation, median, quartiles 1 and 3 and extreme values (minimum and maximum values), and qualitative variables by the frequency and percentage of each response choice with missing data being taken into account as a separate category in the calculation of the percentages. For comparison between preterm and full-term infants, $t$ tests were used for continuous 


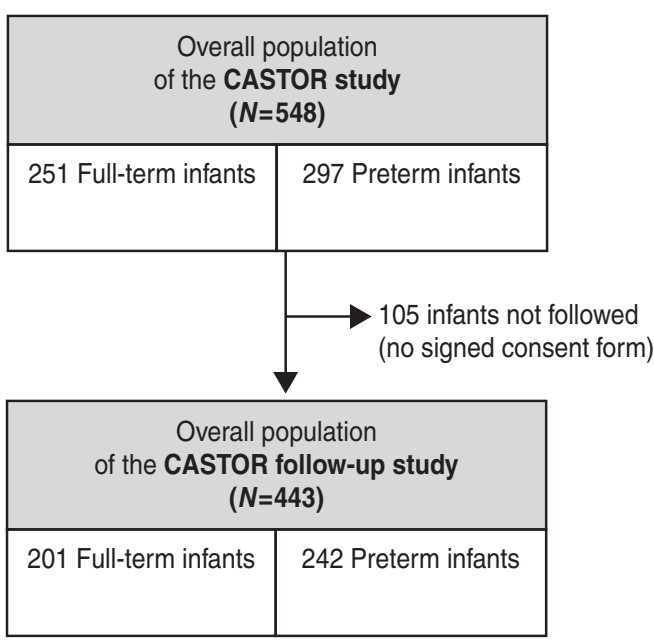

Fig. 2. Disposition of infants.

variables; $\chi^{2}$ or Fisher's exact tests (depending on the standard conditions of application) were used for categorical variables; and Mann-Whitney-Wilcoxon tests were used for continuous variables when the assumptions of the $t$ test were not satisfied (e.g. NSRM counts).

A logistic regression model was used to determine the risk factors for the presence of wheezing or recurrent wheezing episodes during the 12 months following the 2008-2009 RSV season; recurrent wheezing was defined as the occurrence of $\geqslant 3$ episodes of wheezing during the 12-month follow-up period.

The following covariates were first included in univariate logistic regression models: age groups at the beginning of the 2008-2009 RSV season ( $<60$ days, 60-89 days, 90-119 days, 120-149 days, $\geqslant 150$ days); gender; geographical location (region); environmental risk factors (smoking status of the mother during pregnancy, smoking status of the parents since birth, breastfeeding, one or more sibling aged $\geqslant 2$ years, child care); gestational age (preterm infant, full-term infant); birth weight; multiple pregnancy; number of RSV-confirmed bronchiolitis hospitalizations during the first RSV season; number of bronchiolitis hospitalizations irrespective of aetiology during the first RSV season; severity of RSV-confirmed bronchiolitis hospitalizations during the first RSV season (admission to a neonatology unit for at least one bronchiolitis episode, admission to a paediatric intensive care unit for at least one bronchiolitis episode, need of oxygen therapy for at least one bronchiolitis episode, assisted ventilation for at least one bronchiolitis episode); and severity of bronchiolitis hospitalizations irrespective of aetiology during the first RSV season (admission to a neonatology unit for at least one bronchiolitis episode, admission to a paediatric intensive care unit for at least one bronchiolitis episode, need of oxygen therapy for at least one bronchiolitis episode, assisted ventilation for at least one bronchiolitis episode). Only the covariates significant at a threshold of $P \leqslant 0 \cdot 25$ were included in the subsequent logistic multivariate models. A backward selection was used in the final model to retain only those covariates significant at the threshold of $P \leqslant 0 \cdot 05$.

\section{RESULTS}

Of the 548 infants enrolled in the CASTOR study, 443 were followed in the CASTOR follow-up study from June 2009 to June 2010 (Fig. 2); 105 infants (50 fullterm, 55 preterm) were not included in the follow-up study because parents did not sign the consent form. Followed-up infants had similar characteristics compared to those not enrolled in the follow-up, except for a younger mean age (12.5 months vs. 11.7 months, $P<0.001)$ in the non-followed-up group (Table 1). A total of 1985 parents' questionnaires were completed at quarterly phone calls.

\section{Study population}

At inclusion in the CASTOR follow-up study, the mean age of the infants was $12.5 \pm 1.9$ months (range $9 \cdot 0-18 \cdot 0$ months) with the majority being boys $(54 \%)$. Comparative characteristics of the preterm and fullterm groups for the overall population are presented in Table 2.

Preterm infants had significantly lower birth weights and heights, and significantly more were from multiple pregnancies than full-term infants. All preterm infants and 3\% of full-term infants were hospitalized in neonatology units at birth (Table 2). In the preterm group, $35 \%$ of infants $(n=85)$ required mechanical ventilation during the birth hospitalization (mean duration $1.7 \pm 1.3$ days) compared to none of the full-term infants $(P=0.215) ; 68 \%$ of the preterm infants $(n=164)$ had a non-invasive ventilation (mean duration $5 \cdot 7 \pm 6 \cdot 1$ days) in the postnatal period vs. only one full-term infant $(P=0 \cdot 016)$. Regarding environmental risk factors for bronchiolitis, preterm infants were twice as often exposed to antenatal smoking and significantly less breastfed compared to full-terms; conversely, they were one-sixth as often 
Table 1. Comparison of socio-demographic characteristics of patients included and not included in the CASTOR follow-up study $(N=548)$

\begin{tabular}{|c|c|c|c|c|}
\hline Variable & $\begin{array}{l}\text { Included in CASTOR } \\
\text { follow-up } \\
(n=443)\end{array}$ & $\begin{array}{l}\text { Not included in } \\
\text { CASTOR follow-up } \\
(n=105)\end{array}$ & $\begin{array}{l}\text { Total } \\
(N=548)\end{array}$ & $P$ value* \\
\hline \multicolumn{5}{|l|}{ Age (months) $\dagger$} \\
\hline$n$ & 443 & 105 & 548 & \multirow{5}{*}{$<0.001$} \\
\hline Mean (S.D.) & $12 \cdot 5(1 \cdot 9)$ & $11 \cdot 7(1 \cdot 8)$ & $12 \cdot 3(1 \cdot 9)$ & \\
\hline Median & $13 \cdot 0$ & $12 \cdot 0$ & $13 \cdot 0$ & \\
\hline Q1-Q3 & $11 \cdot 0-14 \cdot 0$ & $10 \cdot 0-13 \cdot 0$ & $11 \cdot 0-14 \cdot 0$ & \\
\hline Min-Max & $9 \cdot 0-18 \cdot 0$ & $8 \cdot 0-14 \cdot 0$ & $8 \cdot 0-18 \cdot 0$ & \\
\hline \multicolumn{5}{|l|}{ Sex } \\
\hline Male & $239(54 \cdot 0 \%)$ & $47(44 \cdot 8 \%)$ & $286(52 \cdot 2 \%)$ & \multirow[t]{3}{*}{$0 \cdot 090$} \\
\hline Female & $204(46 \cdot 0 \%)$ & $58(55 \cdot 2 \%)$ & $262(47 \cdot 8 \%)$ & \\
\hline Missing & $0(0 \cdot 0 \%)$ & $0(0 \cdot 0 \%)$ & $0(0 \cdot 0 \%)$ & \\
\hline \multicolumn{5}{|c|}{ Multiple pregnancy } \\
\hline Yes & $99(22 \cdot 3 \%)$ & $16(15 \cdot 2 \%)$ & $115(21 \cdot 0 \%)$ & \multirow[t]{3}{*}{$0 \cdot 161$} \\
\hline No & $340(76 \cdot 7 \%)$ & $87(82 \cdot 9 \%)$ & $427(77 \cdot 9 \%)$ & \\
\hline Missing & $4(0 \cdot 9 \%)$ & $2(1.9 \%)$ & $6(1 \cdot 1 \%)$ & \\
\hline \multicolumn{5}{|c|}{$\begin{array}{l}\text { Gestational age at birth in } \\
\text { preterm group (weeks) }\end{array}$} \\
\hline$n$ & 242 & 55 & 297 & \multirow{5}{*}{0.539} \\
\hline Mean (S.D.) & $31 \cdot 4(0 \cdot 9)$ & $31 \cdot 3(1 \cdot 1)$ & $31 \cdot 3(0 \cdot 9)$ & \\
\hline Median & $32 \cdot 0$ & $32 \cdot 0$ & $32 \cdot 0$ & \\
\hline Q1-Q3 & $31 \cdot 0-32 \cdot 0$ & $31 \cdot 0-32 \cdot 0$ & $31 \cdot 0-32 \cdot 0$ & \\
\hline Min-Max & $28 \cdot 0-32 \cdot 0$ & $28 \cdot 0-32 \cdot 0$ & $28 \cdot 0-32 \cdot 0$ & \\
\hline \multicolumn{5}{|l|}{ Birth weight (g) } \\
\hline$n$ & 443 & 105 & 548 & \multirow{5}{*}{0.955} \\
\hline Mean (S.D.) & $2420 \cdot 7(965 \cdot 3)$ & $2414 \cdot 7(1011 \cdot 5)$ & $2419 \cdot 6(973 \cdot 4)$ & \\
\hline Median & $1980 \cdot 0$ & $2120 \cdot 0$ & $2010 \cdot 0$ & \\
\hline Q1-Q3 & $1580 \cdot 0-3330 \cdot 0$ & $1500 \cdot 0-3400 \cdot 0$ & $1577 \cdot 5-3337 \cdot 5$ & \\
\hline Min-Max & $820 \cdot 0-4490 \cdot 0$ & $900 \cdot 0-4695 \cdot 0$ & $820 \cdot 0-4695 \cdot 0$ & \\
\hline \multicolumn{5}{|c|}{ Birth height (cm) } \\
\hline$n$ & 398 & 96 & 494 & \multirow{5}{*}{0.979} \\
\hline Mean (s.D.) & $44 \cdot 6(5 \cdot 1)$ & $44 \cdot 6(5 \cdot 6)$ & $44 \cdot 6(5 \cdot 2)$ & \\
\hline Median & $43 \cdot 5$ & $43 \cdot 8$ & $43 \cdot 5$ & \\
\hline Q1-Q3 & $40 \cdot 0-50 \cdot 0$ & $9 \cdot 5-50 \cdot 0$ & $40 \cdot 0-50 \cdot 0$ & \\
\hline Min-Max & $27 \cdot 5-54 \cdot 0$ & $34 \cdot 0-54 \cdot 0$ & $27 \cdot 5-54 \cdot 0$ & \\
\hline
\end{tabular}

*P value for between-group comparisons: $t$ test for continuous variables, $\chi^{2}$ test or Fisher's exact test for categorical variables. $\dagger$ Age at the beginning of the CASTOR follow-up study, for the non-included population the date of inclusion is set at 24 June 2009.

kept in day care vs. full-term infants (Table 2). No significant difference between preterm and fullterm infants was observed regarding the family history of allergy or respiratory problems (Table 2).

More preterm infants had been hospitalized for bronchiolitis $(14 \%, n=34$, including 14 patients with RSV-confirmed bronchiolitis) from birth to date of inclusion (not limited to the first RSV season) in the follow-up study than full-term infants $(2 \%, n=4$, including three patients with RSV-confirmed bronchiolitis) $(P<0 \cdot 001)$ (Table 2).

\section{Respiratory morbidities during the 12-month follow-up period}

\section{Wheezing and recurrent wheezing episodes}

Seventy-four infants (23 full-term, 51 preterm) had at least one wheezing episode during the 12-month follow-up period following the first RSV season, 45 had one episode, 17 had two episodes, and 12 had $\geqslant 3$ wheezing episodes (Table 3 ).

Preterm infants were more likely to have at least one wheezing episode than full-term infants: 51/242 
Table 2. Characteristics of the overall CASTOR follow-up population $(N=443)$

\begin{tabular}{|c|c|c|c|}
\hline Variables* & $\begin{array}{l}\text { Full-term infants } \\
(n=201)\end{array}$ & $\begin{array}{l}\text { Preterm infants } \\
(n=242)\end{array}$ & $P$ value $\dagger$ \\
\hline \multicolumn{4}{|l|}{ Socio-demographic characteristics } \\
\hline Age, months & $12 \cdot 4 \pm 1 \cdot 9$ & $12 \cdot 5 \pm 1 \cdot 9$ & \multirow[t]{2}{*}{$0 \cdot 667$} \\
\hline Gestational age at birth, weeks & - & $31 \cdot 4 \pm 0 \cdot 9$ & \\
\hline \multicolumn{4}{|l|}{ Gender } \\
\hline Boys & $108(53 \cdot 7)$ & $131(54 \cdot 1)$ & \multirow[t]{2}{*}{0.933} \\
\hline Girls & $93(46 \cdot 3)$ & $111(45 \cdot 9)$ & \\
\hline From multiple pregnancy: yes & $1(0 \cdot 5)$ & $98(40 \cdot 5)$ & $<0 \cdot 001$ \\
\hline Birth weight, $\mathrm{g}$ & $3403 \cdot 5 \pm 415 \cdot 7$ & $1604 \cdot 4 \pm 301 \cdot 7$ & $<0 \cdot 001$ \\
\hline Birth height, $\mathrm{cm}$ & $50 \cdot 1 \pm 1 \cdot 8$ & $40 \cdot 8 \pm 2 \cdot 7$ & $<0.001$ \\
\hline \multicolumn{4}{|l|}{ Hospital stay after birth } \\
\hline Type of birth centre $\S$ & & & \\
\hline Type 3 & $135(67 \cdot 2)$ & $197(81 \cdot 4)$ & \multirow[t]{4}{*}{$<0 \cdot 001$} \\
\hline Type 2 & $61(30 \cdot 3)$ & $35(14 \cdot 5)$ & \\
\hline Other & $5(2 \cdot 5)$ & $6(2 \cdot 5)$ & \\
\hline Missing & 0 & $4(1 \cdot 6)$ & \\
\hline Total length of stay, days & $5 \cdot 0 \pm 6 \cdot 7$ & $39 \cdot 8 \pm 12 \cdot 5$ & $<0 \cdot 001$ \\
\hline Hospitalization in neonatology units\| & $6(3 \cdot 0)$ & $242(100)$ & $<0 \cdot 001$ \\
\hline Hospitalization for bronchiolitis & $4(2 \cdot 0)$ & $34(14 \cdot 0)$ & $<0.001$ \\
\hline \multicolumn{4}{|l|}{ Environmental risk factors } \\
\hline Smoking status of mother during pregnancy: yes & $26(12 \cdot 9)$ & $61(25 \cdot 2)$ & 0.001 \\
\hline Smoking status in the child's home: yes & $22(10 \cdot 9)$ & $19(7 \cdot 9)$ & $0 \cdot 269$ \\
\hline Number of siblings & $0.9 \pm 0.9$ & $1 \cdot 0 \pm 1 \cdot 0$ & $0 \cdot 084$ \\
\hline Breastfeeding: yes & $136(67 \cdot 7)$ & $133(55 \cdot 0)$ & 0.007 \\
\hline \multicolumn{4}{|l|}{ Child care } \\
\hline Family & $105(52 \cdot 2)$ & $182(75 \cdot 2)$ & \multirow[t]{4}{*}{$<0 \cdot 001$} \\
\hline Child minder & $59(29 \cdot 4)$ & $54(22 \cdot 3)$ & \\
\hline Day-care centre & $21(10 \cdot 4)$ & $4(1 \cdot 7)$ & \\
\hline Other & $16(8 \cdot 0)$ & $2(0 \cdot 9)$ & \\
\hline Family history of allergy\# & $106(52 \cdot 7)$ & $108(44 \cdot 6)$ & $0 \cdot 137$ \\
\hline Family history of respiratory problems\# & $89(44 \cdot 3)$ & $90(37 \cdot 2)$ & $0 \cdot 230$ \\
\hline
\end{tabular}

* Results are presented as meants.D. or $n(\%)$.

$\dagger P$ value for between-group comparisons: $t$ test for continuous variables, $\chi^{2}$ test or Fisher's exact test for categorical variables. $\$$ From birth to going home.

$\S$ Type 2: neonatal intermediate care units; type 3: neonatal intensive care units.

|| Distinct hospital units dedicated to the postnatal management of preterm infants and/or newborns with an altered health status.

I From birth to date of inclusion in the CASTOR follow-up study.

\# No details available as data recorded via parents' questionnaires.

(21\%) versus $23 / 201(11 \%) \quad(P=0.016$ as shown in Table 3). Recurrent wheezing episodes ( $\geqslant 3$ wheezing episodes) concerned $4 \%$ of preterm infants $(10 / 242)$ compared to only $1 \%$ of full-term infants $(2 / 201)$ $(P=0 \cdot 043)$.

According to the history of hospitalized bronchiolitis (irrespective of aetiology) during the first RSV season, $30 \%(11 / 37)$ of the previously hospitalized infants had at least one episode of wheezing during the follow-up period compared to only $15 \cdot 5 \%$
(63/406) of the infants without a previous bronchiolitis hospitalization, irrespective of prematurity status (Table 3).

When considering the cases with a history of hospitalized RSV-confirmed bronchiolitis during the first RSV season, previously hospitalized infants irrespective of their prematurity status were more likely to have at least one wheezing episode than infants with no history of hospitalization [47\% (8/17) vs. $15 \cdot 5 \%$, (66/426)] (Table 4). 


\begin{tabular}{|c|c|c|c|c|c|c|c|c|c|}
\hline \multirow[b]{2}{*}{$\begin{array}{l}\text { Respiratory } \\
\text { morbidity during } \\
12 \text { months following } \\
2008-2009 \text { RSV season }\end{array}$} & \multicolumn{3}{|c|}{ Full-term infants $(n=201)$} & \multicolumn{3}{|c|}{ Preterm infants $(n=242)$} & \multicolumn{3}{|c|}{ Total $(N=443)$} \\
\hline & $\begin{array}{l}\text { History of } \\
\text { hospitalized } \\
\text { bronchiolitis } \\
(n=4)\end{array}$ & $\begin{array}{l}\text { No history of } \\
\text { hospitalized } \\
\text { bronchiolitis } \\
(n=197)\end{array}$ & $\begin{array}{l}\text { Total } \\
\text { full-terms } \\
(n=201)\end{array}$ & $\begin{array}{l}\text { History of } \\
\text { hospitalized } \\
\text { bronchiolitis } \\
(n=33)\end{array}$ & $\begin{array}{l}\text { No history of } \\
\text { hospitalized } \\
\text { bronchiolitis } \\
(n=209)\end{array}$ & $\begin{array}{l}\text { Total } \\
\text { preterms } \\
(n=242)\end{array}$ & $\begin{array}{l}\text { History of } \\
\text { hospitalized } \\
\text { bronchiolitis } \\
(n=37)\end{array}$ & $\begin{array}{l}\text { No history of } \\
\text { hospitalized } \\
\text { bronchiolitis } \\
(n=406)\end{array}$ & $\begin{array}{l}\text { Total } \\
(n=443)\end{array}$ \\
\hline \multicolumn{10}{|l|}{ Number of infants with } \\
\hline No wheezing episode & $2(50 \cdot 0)$ & $176(89 \cdot 3)$ & $178(88 \cdot 6)$ & $24(72 \cdot 7)$ & $167(79 \cdot 9)$ & $191(78 \cdot 9)$ & $26(70 \cdot 3)$ & $343(84 \cdot 5)$ & $369(83 \cdot 3)$ \\
\hline 1 wheezing episode & $2(50 \cdot 0)$ & $14(7 \cdot 1)$ & $16(8)$ & $4(12 \cdot 1)$ & $25(12 \cdot 0)$ & $29(12)$ & $6(16 \cdot 2)$ & $39(9 \cdot 6)$ & $45(10 \cdot 2)$ \\
\hline 2 wheezing episodes & - & $5(2 \cdot 5)$ & $5(2 \cdot 5)$ & $3(9 \cdot 1)$ & $9(4 \cdot 3)$ & $12(5)$ & $3(8 \cdot 1)$ & $14(3 \cdot 4)$ & $17(3 \cdot 8)$ \\
\hline 3 wheezing episodes & - & $2(1 \cdot 0)$ & $2(1)$ & $1(3 \cdot 0)$ & $6(2 \cdot 9)$ & $7(2 \cdot 9)$ & $1(2 \cdot 7)$ & $8(2 \cdot 0)$ & $9(2 \cdot 0)$ \\
\hline$>3$ wheezing episodes & - & - & - & $1(3 \cdot 0)$ & $2(1 \cdot 0)$ & $3(1 \cdot 2)$ & $1(2 \cdot 7)$ & $2(0 \cdot 5)$ & $3(0 \cdot 7)$ \\
\hline \multicolumn{10}{|l|}{ Number of infants with } \\
\hline At least one episode of NSRM & $2(50 \cdot 0)$ & $46(23 \cdot 4)$ & $48(23 \cdot 9)$ & $18(54 \cdot 5)$ & $62(29 \cdot 7)$ & $80(33 \cdot 1)$ & $20(54 \cdot 1)$ & $108(26 \cdot 6)$ & $128(28 \cdot 9)$ \\
\hline No episode of NSRM & $2(50 \cdot 0)$ & $151(76 \cdot 6)$ & $153(76 \cdot 1)$ & $15(45 \cdot 5)$ & $147(70 \cdot 3)$ & $162(66 \cdot 9)$ & $17(45 \cdot 9)$ & $298(73 \cdot 4)$ & $315(71 \cdot 1)$ \\
\hline \multicolumn{10}{|l|}{ Hospitalization for wheezing } \\
\hline Yes & - & - & - & - & $2(1 \cdot 0)$ & $2(0 \cdot 8)$ & - & $2(0 \cdot 5)$ & $2(0 \cdot 5)$ \\
\hline No & $4(100)$ & $197(100)$ & $201(100)$ & $33(100)$ & $207(99 \cdot 0)$ & $240(99 \cdot 2)$ & $37(100)$ & $404(99 \cdot 5)$ & $441(99 \cdot 5)$ \\
\hline \multicolumn{10}{|l|}{ Hospitalization for bronchiolitis* } \\
\hline Yes & - & - & - & - & $1(0 \cdot 5)$ & $1(0 \cdot 4)$ & - & $1(0 \cdot 2)$ & $1(0 \cdot 2)$ \\
\hline No & $4(100)$ & $197(100)$ & $201(100)$ & $33(100)$ & $208(99 \cdot 5)$ & $241(99 \cdot 6)$ & $37(100)$ & $405(99 \cdot 8)$ & $442(99 \cdot 8)$ \\
\hline \multicolumn{10}{|l|}{$\begin{array}{l}\text { Hospitalization for other respiratory } \\
\text { morbidity problems }\end{array}$} \\
\hline Yes & - & $1(0 \cdot 5)$ & $1(0 \cdot 5)$ & - & $1(0 \cdot 5)$ & $1(0 \cdot 4)$ & - & $2(0 \cdot 5)$ & $2(0 \cdot 5)$ \\
\hline No & $4(100)$ & $196(99 \cdot 5)$ & $200(99 \cdot 5)$ & $33(100)$ & $208(99 \cdot 5)$ & $241(99 \cdot 6)$ & $37(100)$ & $404(99 \cdot 5)$ & $441(99 \cdot 5)$ \\
\hline
\end{tabular}

hospitalization irrespective of aetiology during the 2008-2009 RSV season $(N=443)$

RSV, Respiratory syncytial virus; NSRM, non-specified respiratory morbidity.

Data are presented as $n(\%)$.

* All were for RSV bronchiolitis.

All were ror Rv bronchiolitis. 
Table 4. Disposition of respiratory morbidities during the 12 months following the 2008-2009 RSV season according to the history of RSV-confirmed bronchiolitis hospitalization during the 2008-2009 RSV season $(N=443)$

\begin{tabular}{|c|c|c|c|c|c|c|c|c|c|}
\hline \multirow[b]{2}{*}{$\begin{array}{l}\text { Respiratory } \\
\text { morbidity during } \\
12 \text { months following } \\
2008-2009 \text { RSV } \\
\text { season }\end{array}$} & \multicolumn{3}{|c|}{ Full-term infants $(n=201)$} & \multicolumn{3}{|c|}{ Preterm infants $(n=242)$} & \multicolumn{3}{|c|}{ Total $(N=443)$} \\
\hline & $\begin{array}{l}\text { History of } \\
\text { hospitalized } \\
\text { RSV } \\
\text { bronchiolitis } \\
(n=3)\end{array}$ & $\begin{array}{l}\text { No history of } \\
\text { hospitalized } \\
\text { RSV } \\
\text { bronchiolitis } \\
(n=198)\end{array}$ & $\begin{array}{l}\text { Total } \\
\text { full-terms } \\
(n=201)\end{array}$ & $\begin{array}{l}\text { History of } \\
\text { hospitalized } \\
\text { RSV } \\
\text { bronchiolitis } \\
(n=14)\end{array}$ & $\begin{array}{l}\text { No history of } \\
\text { hospitalized } \\
\text { RSV } \\
\text { bronchiolitis } \\
(n=228)\end{array}$ & $\begin{array}{l}\text { Total } \\
\text { preterms } \\
(n=242)\end{array}$ & $\begin{array}{l}\text { History of } \\
\text { hospitalized } \\
\text { RSV } \\
\text { bronchiolitis } \\
(n=17)\end{array}$ & $\begin{array}{l}\text { No history of } \\
\text { hospitalized } \\
\text { RSV } \\
\text { bronchiolitis } \\
(n=426)\end{array}$ & $\begin{array}{l}\text { Total } \\
(N=443)\end{array}$ \\
\hline \multicolumn{10}{|l|}{ Number of infants with } \\
\hline No wheezing episode & $1(33 \cdot 3)$ & $177(89 \cdot 4)$ & $178(88 \cdot 6)$ & $8(57 \cdot 1)$ & $183(80 \cdot 3)$ & $191(78 \cdot 9)$ & $9(52 \cdot 9)$ & $360(84 \cdot 5)$ & $369(83 \cdot 3)$ \\
\hline 1 wheezing episode & $2(66 \cdot 7)$ & $14(7 \cdot 1)$ & $16(8)$ & $3(21 \cdot 4)$ & $26(11 \cdot 4)$ & $29(12)$ & $5(29 \cdot 4)$ & $40(9 \cdot 4)$ & $45(10 \cdot 2)$ \\
\hline 2 wheezing episodes & - & $5(2 \cdot 5)$ & $5(2 \cdot 5)$ & $2(14 \cdot 3)$ & $10(4 \cdot 4)$ & $12(5)$ & $2(11 \cdot 8)$ & $15(3 \cdot 5)$ & $17(3 \cdot 8)$ \\
\hline 3 wheezing episodes & - & $2(1 \cdot 0)$ & $2(1)$ & $1(7 \cdot 1)$ & $6(2 \cdot 6)$ & $7(2 \cdot 9)$ & $1(5 \cdot 9)$ & $8(1 \cdot 9)$ & $9(2 \cdot 0)$ \\
\hline$>3$ wheezing episodes & - & - & - & - & $3(1 \cdot 3)$ & $3(1 \cdot 2)$ & - & $3(0 \cdot 7)$ & $3(0 \cdot 7)$ \\
\hline \multicolumn{10}{|l|}{ Number of infants with } \\
\hline At least one episode of NSRM & $1(33 \cdot 3)$ & $47(23 \cdot 7)$ & $48(23 \cdot 9)$ & $11(78 \cdot 6)$ & $69(30 \cdot 3)$ & $80(33 \cdot 1)$ & $12(70 \cdot 6)$ & $116(27 \cdot 2)$ & $128(28 \cdot 9)$ \\
\hline No episode of NSRM & $2(66 \cdot 7)$ & $151(76 \cdot 3)$ & $153(76 \cdot 1)$ & $3(21 \cdot 4)$ & $159(69 \cdot 7)$ & $162(66 \cdot 9)$ & $5(29 \cdot 4)$ & $310(72 \cdot 8)$ & $315(71 \cdot 1)$ \\
\hline \multicolumn{10}{|l|}{ Hospitalization for wheezing } \\
\hline Yes & - & - & - & - & $2(0 \cdot 9)$ & $2(0 \cdot 8)$ & - & $2(0 \cdot 5)$ & $2(0 \cdot 5)$ \\
\hline No & $3(100)$ & $198(100)$ & $201(100)$ & $14(100)$ & $226(99 \cdot 1)$ & $240(99 \cdot 2)$ & $17(100)$ & $424(99 \cdot 5)$ & $441(99 \cdot 5)$ \\
\hline \multicolumn{10}{|l|}{ Hospitalization for bronchiolitis* } \\
\hline Yes & - & - & - & - & $1(0 \cdot 4)$ & $1(0 \cdot 4)$ & - & $1(0 \cdot 2)$ & $1(0 \cdot 2)$ \\
\hline No & $3(100)$ & $198(100)$ & $201(100)$ & $14(100)$ & $227(99 \cdot 6)$ & $241(99 \cdot 6)$ & $17(100)$ & $425(99 \cdot 8)$ & $442(99 \cdot 8)$ \\
\hline \multicolumn{10}{|l|}{$\begin{array}{l}\text { Hospitalization for other respiratory } \\
\text { problems }\end{array}$} \\
\hline Yes & - & $1(0 \cdot 5)$ & $1(0 \cdot 5)$ & - & $1(0 \cdot 4)$ & $1(0 \cdot 4)$ & - & $2(0 \cdot 5)$ & $2(0 \cdot 5)$ \\
\hline No & $3(100)$ & $197(99 \cdot 5)$ & $200(99 \cdot 5)$ & $14(100)$ & $227(99 \cdot 6)$ & $241(99 \cdot 6)$ & $17(100)$ & $424(99 \cdot 5)$ & $441(99 \cdot 5)$ \\
\hline
\end{tabular}

RSV, Respiratory syncytial virus; NSRM, non-specified respiratory morbidity.

Data are presented as $n(\%)$.

* All were for RSV bronchiolitis. 


\section{NSRM}

A total of $128(28.9 \%)$ infants had at least one episode of NSRM (Table 5). Preterm infants were significantly $(P=0.034)$ more likely to have at least one episode of NSRM than full-terms, and had significantly $(P=0.042)$ more frequent NSRM episodes compared to full-term infants as shown in Table 5 .

According to the history of hospitalized bronchiolitis (irrespective of aetiology) during the first RSV season, previously hospitalized infants were more likely to have at least one episode of NSRM than those without a previous bronchiolitis hospitalization [54\% (20/37) vs. 27\% (108/406)], irrespective of prematurity status (Table 3). When analysing the impact of a RSV-confirmed bronchiolitis hospitalization during the first RSV season, previously hospitalized infants were more likely to have at least one episode of NSRM during the follow-up period than those with no history [71\% (12/17) vs. 27\% (116/426)], irrespective of their prematurity status (Table 4).

\section{Hospitalizations for respiratory pathologies}

Five infants (four preterm, one full-term) were hospitalized during the 12-month follow-up period. In the preterm group, the reasons for hospitalization were: RSV bronchiolitis $(n=1)$, wheezing $(n=2)$, and dyspnoea related to oesogastric reflux $(n=1)$; the fullterm infant was hospitalized for a right basal pneumopathy.

All the hospitalizations for respiratory problems occurred in infants without a history of bronchiolitis hospitalization as shown in Table 3.

\section{Predictive factors of wheezing and recurrent wheezing episodes during the 12-month follow-up period}

The following factors were found to be linked to the occurrence of wheezing episodes at the threshold of $P<0.25$ and were included in the multivariate logistic model: gender $(P=0.022)$, smoking status of the parents since birth to inclusion in the CASTOR study $(P=0 \cdot 172)$, breastfeeding $(P=0 \cdot 197)$, prematurity $(P=0.023)$, birth weight $(P=0.046)$, RSVconfirmed bronchiolitis hospitalization during the first RSV season $(P=0.002)$, and need of oxygen therapy for an RSV-confirmed bronchiolitis hospitalization during the first RSV season $(P=0 \cdot 006)$. A backward selection was used to retain only those factors that were significant at the threshold of 5\% in the final model. As shown in Table 6, three covariates were statistically significant in the final model, indicating that the probability of having wheezing episodes during the 12-month period following the first RSV season was significantly impacted by these three independent factors: male gender, birth weight $<3330 \mathrm{~g}$, and hospitalization for RSVconfirmed bronchiolitis during the first RSV season.

Regarding recurrent wheezing episodes during the 12-month follow-up period, no independent predictive factors of such episodes were identified in the final multivariate model.

\section{DISCUSSION}

The main findings of the CASTOR follow-up study are: (i) preterm infants $<33$ weeks GA without BPD had a greater respiratory morbidity (wheezing episodes or NSRM episodes) than full-term infants; (ii) infants with a history of hospitalized bronchiolitis (RSV confirmed and all types) during their first RSV season were more likely to have at least one episode of wheezing or NSRM during the subsequent 12 months than infants without such a history; (iii) RSV-confirmed bronchiolitis hospitalization during their first RSV season, birth weight $<3330 \mathrm{~g}$ and male gender emerged as independent risk factors for wheezing episodes during the subsequent 12 months.

The CASTOR follow-up study provides prospective data on the chronic respiratory morbidity in a large French cohort of 443 infants (out of which 242 preterm infants $<33$ weeks GA without BPD) followed up during the 12 months following their first RSV season. To our knowledge, only one previous prospective study evaluated post-RSV respiratory morbidity and that was in a smaller cohort of 126 preterm infants $<32$ weeks GA followed up to only age 1 year of [32].

The main purpose of the CASTOR follow-up study was to collect data on the occurrence of wheezing and recurrent wheezing episodes during the 12 months following the 2008-2009 RSV season in infants from the CASTOR cohort. The comparison between the preterm group ( $<33$ weeks GA) without BPD and the full-term group (39-41 weeks GA) highlighted the impact of premature birth $(<33$ weeks GA) on the increased incidence of wheezing episodes during the 12-month follow-up period after their first RSV season, even in the absence of an associated chronic lung disease.

Hoo et al. [29] showed via paired measurements of maximal expiratory flow at functional residual capacity, obtained 3 weeks after birth in 24 preterm 
Table 5. Description of non-specified respiratory morbidity during the 12 months following the 2008-2009 RSV season in the overall CASTOR follow-up population $(N=443)$

\begin{tabular}{lcclc}
\hline \hline & $\begin{array}{l}\text { Full-term } \\
\text { infants } \\
(n=201)\end{array}$ & $\begin{array}{l}\text { Preterm } \\
\text { infants } \\
(n=242)\end{array}$ & $\begin{array}{l}\text { Total } \\
(N=443)\end{array}$ & $P$ value \\
\hline Number of infants with & & & & $0 \cdot 034^{*}$ \\
At least one episode of NSRM & $48(23 \cdot 9)$ & $80(33 \cdot 1)$ & $128(28 \cdot 9)$ & \\
No episode of NSRM & $153(76 \cdot 1)$ & $162(66 \cdot 9)$ & $315(71 \cdot 1)$ & \\
Number of infants with & & & & $0.042 \dagger$ \\
No episode of NSRM & $153(76 \cdot 1)$ & $162(66 \cdot 9)$ & $315(71 \cdot 1)$ & \\
1 episode of NSRM & $28(13 \cdot 9)$ & $49(20 \cdot 2)$ & $77(17 \cdot 4)$ & \\
2 episodes of NSRM & $12(6 \cdot 0)$ & $18(7 \cdot 4)$ & $30(6 \cdot 8)$ & \\
3 episodes of NSRM & $4(2 \cdot 0)$ & $9(3 \cdot 7)$ & $13(2 \cdot 9)$ & \\
$>3$ episodes of NSRM & $4(2 \cdot 0)$ & $4(1 \cdot 7)$ & $8(1 \cdot 8)$ & \\
\hline \hline
\end{tabular}

RSV, Respiratory syncytial virus; NSRM, non-specified respiratory morbidity.

Data are presented as $n(\%)$.

$* \chi^{2}$ test.

$\dagger$ Mann-Whitney-Wilcoxon test.

Table 6. Predictive factors of wheezing episodes that occurred during the 12 months following the 2008-2009 RSV season in the overall CASTOR follow-up population $(N=443)$

\begin{tabular}{llll}
\hline \hline Covariates* & & OR $(95 \% \mathrm{CI})$ & $P$ value \\
\hline Sex (ref. = female) & Male & $2 \cdot 11(1 \cdot 23-3 \cdot 64)$ & $0 \cdot 007$ \\
Birth weight (ref.=3330-4490 g) & & & $0 \cdot 018 \dagger$ \\
& $820-<1580 \mathrm{~g}$ & $3 \cdot 21(1 \cdot 38-7 \cdot 49)$ & \\
& $1580-<1980 \mathrm{~g}$ & $2 \cdot 61(1 \cdot 11-6 \cdot 14)$ & \\
& $1980-<3330 \mathrm{~g}$ & $3 \cdot 76(1 \cdot 61-8 \cdot 77)$ & \\
& Yes & $4 \cdot 72(1 \cdot 71-13 \cdot 08)$ & $0 \cdot 003$ \\
RSV-confirmed bronchiolitis + & & & \\
(ref.= no) & & & \\
\hline \hline
\end{tabular}

RSV, Respiratory syncytial virus; OR, odds ratio; CI, confidence interval.

* Covariates included in the multivariate logistic regression model: gender, smoking status of the parents since birth to inclusion in the CASTOR study, breastfeeding, prematurity, birth weight, RSV-confirmed bronchiolitis hospitalization during the first RSV season, and need of oxygen therapy for an RSV-confirmed bronchiolitis hospitalization during the first RSV season.

$\dagger 820-<3330$ g vs. $3330-4490 \mathrm{~g}$.

$\$$ Hospitalization for RSV-confirmed bronchiolitis during the first RSV season.

infants (mean GA $33 \cdot 2 \pm 2 \cdot 2$ weeks), and repeated at a mean corrected postnatal age of $57 \cdot 0 \pm 12 \cdot 2$ weeks, that preterm delivery ( $\leqslant 36$ weeks GA) was associated with altered airway development during early infancy even in the absence of neonatal respiratory disease. Friedrich et al. [30] previously showed persistently reduced expiratory flows in the presence of normal forced vital capacity and the absence of catch-up growth in airway function based on longitudinal measurements of forced expiratory flow obtained in the first and the second years of life for 26 preterms born on average at 32.7 (range 30-34) weeks' GA $(n=26)$ compared to 24 full-terms.

Our findings are in line with previous data on the relationship between early severe bronchiolitis and subsequent recurrent childhood wheezing, a long lasting reduction in pulmonary function, and asthma $[7,9,13,17]$. Despite the higher incidence of wheezing 
(including recurrent wheezing) episodes during the 12-month period following the first RSV season in the overall CASTOR follow-up population, i.e. $11 / 37(30 \%)$ infants with a history of hospitalized bronchiolitis compared to $63 / 406(15 \cdot 5 \%)$ without such a history, we could not show a significant difference between these two groups given the small sample sizes.

Furthermore, infants with a history of early hospitalized RSV-confirmed bronchiolitis were more prone to at least one wheezing episode than those with no such history $(47 \%$ vs. $15 \cdot 5 \%)$, irrespective of their prematurity status, during the CASTOR follow-up period. Our results are consistent with previously published data [38, 39], but with lower incidences than those found by Sigurs et al. in 3-year-old children with RSV bronchiolitis during the first year of life $(60 \%$ of infants with RSV history with any wheezing episodes $v s .32 \%$ of those with no history, $P=0.003$ ) [12]; those authors confirmed the same tendency in this birth cohort up to the age of 7 years [13]. The ALSPAC Study Team showed a lower prevalence of any wheezing (28\%) at 30-42 months after birth in the RSV subgroup (150 infants) of this large British birth cohort compared to our results $(47 \%)$, despite a very close prevalence in controls (13\% and $15.5 \%$, respectively). A likely explanation for this observation is the increased loss of follow-up in the ALSPAC cohort infants with associated risk factors for RSV hospitalization, such as low birth weight, preterm delivery, housing and tobacco smoke exposure [8]. Broughton et al. [32] equally showed that early severe RSV disease in preterm infants had an impact on the subsequent respiratory morbidity and healthcare resources utilization, as preterm infants with a history of RSV infection had significantly more days of cough and wheeze at 1-year follow-up compared to those without LRTI or with RSV-negative LRTI [32].

Our study confirms that early severe RSV bronchiolitis has a deleterious impact on clinical respiratory outcomes of infants followed-up during the 12 months subsequent to their first RSV season. Moreover, early severe viral LRTI in preterm infants has been shown to be associated with abnormal lung function at longterm follow-up [35]. As suggested by Sigurs et al. based on the 18-year follow-up of a cohort of 138 children, out of which 46 had RSV bronchiolitis during their first year of life, the underlying mechanism of subsequent chronic respiratory morbidity could be the airways remodelling [15]. This hypothesis is supported by extensive experimental data showing that
RSV infection induces cellular immune responses with subsequent production of inflammatory mediators that may cause tissue remodelling, with longterm effects on the lung structure and clinical outcomes [40, 41].

Hospitalization for RSV bronchiolitis during the first RSV season was the most important risk factor for the development of subsequent wheezing episodes in the present study; infants with a history of early severe RSV bronchiolitis were 4.72 times as likely to develop wheezing episodes during the 12 months following their first RSV season than their nonhospitalized counterparts.

Our findings are equally supported by a recently published retrospective study based on a large (nearly 14 million members) US health plan database, showing that preterm infants with early severe RSV bronchiolitis were $2 \cdot 52$-fold ( $95 \%$ confidence interval $1 \cdot 65-3 \cdot 85, P<0 \cdot 001)$ as likely to develop early serious childhood wheezing by age 3 years [34].

The CASTOR follow-up study results should be interpreted in the light of several methodological limitations: (i) the study sample size was not determined by statistical considerations but only determined by the participation rate in the follow-up period, thereby imposing a cautious interpretation of the results; (ii) the main purpose of this study was to evaluate the relationship between bronchiolitis hospitalizations during the first RSV season and the occurrence of respiratory morbidities during the following 12 months in the overall follow-up population (larger sample size enabling more robust results); (iii) RSV testing in case of hospitalization for bronchiolitis was not required by the protocol (observational study) and therefore the incidence of RSV hospitalizations could have been underestimated, thus weakening the observed association between early severe RSV-confirmed bronchiolitis and subsequent wheezing. Moreover, while we found that a birth weight $<3330$ g was associated with a higher risk of respiratory morbidity after the first RSV season, this threshold was derived from a statistical approach, and further exploration is needed to strengthen this finding and assess its clinical relevance, in particular using the generally accepted clinical standards for low birth weight.

A potential underestimation of the incidence of wheezing/recurrent wheezing during the follow-up period in our study could have been also determined by: (i) the considerable number of infants who did not participate in the follow-up $(19 \%$ of the overall 
CASTOR study population), especially as these infants were younger than those followed-up; (ii) the lack of diagnosis confirmation by a physician, with potential data loss (presence of wheezing/recurrent wheezing cases in infants diagnosed with other LRTI unknown diagnosis).

\section{CONCLUSION}

In conclusion, the present study demonstrated an increased incidence of respiratory morbidity during the 12 months subsequent to the first RSV season (2008-2009 RSV season) in preterm infants ( $<33$ weeks GA) without associated BPD compared to full-term infants, as well as in infants with a history of early severe bronchiolitis (RSV confirmed and all types) hospitalization compared to those without such a history.

Three factors were predictive of wheezing episodes during the 12 months following the first RSV season, irrespective of prematurity status: early hospitalization for RSV-confirmed bronchiolitis, birth weight $<3330 \mathrm{~g}$, and male gender. The awareness of these risk factors could facilitate the identification of infants at high risk of respiratory morbidities, with the subsequent implementation of more rigorous prophylactic measures.

\section{ACKNOWLEDGEMENTS}

We are extremely grateful to the children and parents who contributed to this study. We thank all investigators and regional coordinators who participated in the study. We thank Brigitte Bourdillat for the manuscript editing. We acknowledge Muriel Viala-Danten, Fatoumata Fofana and Antoine Regnault from MAPI Consultancy for their contribution to the design of statistical analyses and the interpretation of study results. We also thank ProClinica for the logistic assistance in the phone call follow-up with the parents of enrolled children. This study was sponsored by AbbVie France.

\section{DECLARATION OF INTEREST}

B.F. has received honorarium for her scientific advice and expertise from AbbVie. J.B.G. has served as a consultant for AbbVie, Chiesi Laboratories, Orphan Europe Laboratories, and has received research funding from AbbVie, Chiesi Laboratories and Orphan Europe Laboratories. J.C.R. has served as a consultant for AbbVie. D.P. has received honoraria from pharmaceutical companies including AbbVie, GSK, Novartis, Sanofi Pasteur MSD and Wyeth for educational presentations and congress presentations. D.A. and T.M. are employees of AbbVie, and may own AbbVie stock. The design, study conduct, and financial support for the clinical trial was provided by AbbVie. AbbVie participated in the interpretation of data, review, and approval of the manuscript.

\section{REFERENCES}

1. Agence Nationale d'Accréditation et d'Evaluation en Santé (ANAES). Consensus conference. Management of bronchiolitis in infants, 2000 (http://www.has-sante. fr/portail/upload/docs/application/pdf/Bronchiolitis.pdf).

2. Wainwright C. Acute viral bronchiolitis in children $-\mathrm{a}$ very common condition with few therapeutic options. Paediatric Respiratory Reviews 2010; 11: 39-45.

3. Grimprel E. Epidemiology of infant bronchiolitis in France [in French]. Archives de Pédiatrie 2001; 8: 83-92.

4. Busch A, Thomson AH. Acute bronchiolitis. British Medical Journal 2007; 335: 1037-1041.

5. Marguet C, et al. In very young infants severity of acute bronchiolitis depends on carried viruses. PLoS One. Published online: 25 February 2009. doi:10.1371/journal. pone.0004596.

6. Calvo C, et al. Detection of new respiratory viruses in hospitalized infants with bronchiolitis: a three-year prospective study. Acta Paediatrica 2010; 99: 883-887.

7. Jartti T, et al. The link between bronchiolitis and asthma. Infectious Disease Clinics of North America 2005; 19: $667-689$.

8. Henderson $\mathbf{J}$, et al. Hospitalization for RSVbronchiolitis before 12 months of age and subsequent asthma, atopy and wheeze: a longitudinal birth cohort study. Pediatric Allergy and Immunology 2005; 16: 386-392.

9. Fjaerli HO, et al. Acute bronchiolitis in infancy as risk factor for wheezing and reduced pulmonary function by seven years in Akershus County, Norway. BMC Pediatrics. Published online: 18 August 2005. doi:10.1186/1471-2431-5-31.

10. Halfhide C, Smyth RL. Innate immune response and bronchiolitis and preschool recurrent wheeze. Paediatric Respiratory Reviews 2008; 9: 251-262.

11. Stein RT, et al. Respiratory syncytial virus in early life and risk of wheeze and allergy by age 13 years. Lancet 1999; 354: 541-545.

12. Sigurs N, et al. Asthma and immunoglobulin E antibodies after respiratory syncytial virus bronchiolitis: a prospective cohort study with matched control. Pediatrics 1995; 95: 500-505.

13. Sigurs N, et al. Respiratory syncytial virus bronchiolitis in infancy is an important risk factor for asthma and allergy at age 7. American Journal of Respiratory Critical Care Medicine 2000; 161: 1501-1507.

14. Sigurs N, et al. Severe respiratory syncytial virus bronchiolitis in infancy and asthma and allergy at 
age 13. American Journal of Respiratory Critical Care Medicine 2005; 171: 137-141.

15. Sigurs N, et al. Asthma and allergy patterns over 18 years after severe RSV-bronchiolitis in the first year of life. Thorax 2010; 65: 1045-1052.

16. Stensballe LG, et al. The causal direction in the association between respiratory syncytial virus hospitalization and asthma. Journal of Allergy and Clinical Immunology 2009; 123: 131-137.

17. Singh AM, et al. Bronchiolitis to asthma. A review and call for studies of gene-virus interactions in asthma causation. American Journal of Respiratory Critical Care Medicine 2007; 175: 108-119.

18. Thomsen SF, et al. Exploring the association between severe respiratory syncytial virus infection and asthma. A registry-based twin study. American Journal of Respiratory Critical Care Medicine 2009; 179: 1091-1097.

19. Lemanske RF, et al. Rhinovirus illnesses during infancy predict subsequent childhood wheezing. Journal of Allergy and Clinical Immunology 2005; 116: 571-577.

20. Calvo C, et al. Role of rhinovirus in hospitalized infants with respiratory tract infections in Spain. Pediatric Infectious Disease Journal 2007; 26: 904-908.

21. Hyvärinen M, Korppi M. Viral bronchiolitis and asthma development: lessons from longitudinal studies. Current Respiratory Medicine Reviews 2011; 7: 196-202.

22. Jartti T, Korppi M. Rhinovirus-induced bronchiolitis and asthma development. Pediatric Allergy and Immunology 2011; 22: 350-355.

23. Mansbach JM, Camargo CA. Respiratory viruses in bronchiolitis and their link to recurrent wheezing and asthma. Clinical Laboratory Medicine 2009; 29: 741-755.

24. Valkonen H, et al. Recurrent wheezing after respiratory syncytial virus or non-respiratory syncytial virus bronchiolitis in infancy: a 3-year follow-up. Allergy 2009; 64: 1359-1365.

25. Greenough A, Broughton S. Chronic manifestations of respiratory syncytial virus infection in premature infants. Pediatric Infectious Disease Journal 2005; 24: S184-S188.

26. Fleming DM, et al. Respiratory illness associated with influenza and respiratory syncytial virus infection. Archives of Disease in Childhood 2005; 90: 741-746.

27. Stein RT. Long-term airway morbidity following viral LRTI in early infancy: recurrrent wheezing or asthma? Paediatric Respiratory Reviews 2009; 10 (Suppl. 1): 29-31.
28. Welliver RC. Review of epidemiology and clinical risk factors for severe respiratory syncytial virus (RSV) infection. Journal of Pediatrics 2003; 143: S112-S117.

29. Hoo AF, et al. Development of airway function in infancy after preterm delivery. Journal of Pediatrics 2002; 141: 652-658.

30. Friedrich L, et al. Growth rate of lung function in healthy preterm infants. American Journal of Respiratory Critical Care Medicine 2007; 176: 1269-1273.

31. Pinquier D, et al. Palivizumab immunoprophylaxis: use in clinical practice, safety and beneficial effects in France. Archives de Pédiatrie 2009; 16: 1443-1452.

32. Broughton S, et al. Prospective study of healthcare utilisation and respiratory morbidity due to RSV infection in prematurely born infants. Thorax 2005; 60: 1039 1044.

33. Van Bever H. Determinants in early life for asthma development. Allergy, Asthma \& Clinical Immunology 2009; 5: 6.

34. Romero JR, et al. Serious early childhood after respiratory syncytial virus lower respiratory tract illness in preterm infants. Clinical Therapeutics 2010; 32: 2422-2432.

35. Broughton S, et al. Lung function in prematurely born infants after viral lower respiratory tract infections. Pediatric Infectious Disease Journal 2007; 26: 1019 1024.

36. Greenough A. Late respiratory outcomes after preterm birth. Early Human Development 2007; 83: 785-788.

37. Gouyon JB, et al. Hospitalizations for respiratory syncytial virus bronchiolitis in preterm infants at $<33$ weeks gestation without bronchopulmonary dysplasia: the CASTOR study. Epidemiology and Infection 2013; 141: 816-826.

38. Kneyber MCJ, et al. Long-term effects of respiratory syncytial virus (RSV) bronchiolitis in infants and young children: a quantitative review. Acta Paediatrica 2000; 89: 654-660.

39. Osundwa VM, Dawod Ehlayel M. Recurrent wheezing in children with respiratory syncytial virus (RSV) bronchiolitis in Qatar. European Journal of Pediatrics 1993; 152: 1001-1003.

40. Mohapatra SS, Boyapalle S. Epidemiologic, experimental, and clinical links between respiratory syncytial virus infection and asthma. Clinical Microbiology Reviews 2008; 21: 495-504.

41. Martin JG, Siddiqui S, Hassan M. Immune responses to viral infections: relevance for asthma. Paediatric Respiratory Reviews 2006; 75: S125-S127. 